\title{
No se suspende: Explotación Sexual Comercial de la Niñez y la Adolescencia en Tiempos de Capital Pandémico en la realidad brasileña y paraguaya
}

\section{No cancellation: Commercial Sexual Explotation of Children and Adolescents in times of Pandemic Capital in the brazilian and paraguayan reality}

\author{
Alan de Loiola Alves ${ }^{1}$ (
}

\begin{abstract}
Resumen
El objetivo de este artículo es el de reflexionar sobre la explotación sexual comercial de la niñas, niños y adolescentes en Brasil y en Paraguay en tiempos de capital pandémico, específicamente definir qué es explotación sexual de niñas, niños y adolescentes, conocer las características de esta manifestación de la "cuestión social" en Brasil y en Paraguay. La metodología utilizada es mediante la teoría social crítica y recurriendo a una investigación exploratoria seguida de relevamiento bibliográfico y documental. La explotación sexual comercial infanto-juvenil es una manifestación de la "cuestión social" presente en todo el territorio brasileño y paraguayo, saltando las fronteras territoriales, ya que el mercado del sexo es internacional. La pandemia de COVID-19 y la crisis estructural del capital agravaron las desigualdades sociales y aumentaron la vulnerabilidad de niñas, niños y adolescentes delante de la explotación sexual comercial, destacando que el mercado del sexo no paró sus actividades. Esta realidad exige medidas de enfrentamiento por parte del Estado; sin embargo, su supresión exige la eliminación de todas las formas de desigualdad de este orden social, haciéndose necesario construir un nuevo modelo de sociedad.
\end{abstract}

Palabras clave: explotación sexual comercial de niños y adolescentes,cuestión social,capital pandémico.

\begin{abstract}
The aim of the present paper is to foster reflection on the commercial sexual exploitation of children and adolescents in Brazil and Paraguay at times of pandemic capital; more specifically, to define what is sexual exploitation of children and adolescents, to explain the features of this manifestation of "the social question" in Brazil and Paraguay. The approach has been that of critical social theory and the method that of exploratory research plus documentary and bibliographical research. Commercial sexual exploitation of children and adolescents is a manifestation of "the social issue" present throughout the Brazilian and Paraguayan territory, transcending national borders as the sex market is international in nature. The
\end{abstract}

\author{
1 Pontificia Universidad Católica \\ de São Paulo. São Paulo, SP, \\ Brasil. \\ Correspondencia: \\ alanloiola@yahoo.com.br \\ Recibido: \\ 2 de agosto de 2021 \\ Aceptado: \\ 26 de octubre de 2021 \\ Doi:
}

https://doi.org/10.54549/ky.6e.2021.39

\section{cc) (i)}

Artículo publicado en acceso abierto bajo la Licencia Creative Commons.

$$
\begin{aligned}
& \text { Cita: } \\
& \text { Alves, A. L. (2021). No se } \\
& \text { suspende: Explotación } \\
& \text { Sexual Comercial de la } \\
& \text { Niñez y la Adolescencia } \\
& \text { en Tiempos de Capital } \\
& \text { Pandémico en la realidad } \\
& \text { brasileña y paraguaya. Kera } \\
& \text { Yvoty: reflexiones sobre la } \\
& \text { cuestión social, 6(número } \\
& \text { especial), 39-53. https://doi. } \\
& \text { org/10.54549/ky.6e.2021.39 }
\end{aligned}
$$


COVID-19 pandemic and the structural crisis of capital have deepened social inequality and increased the vulnerability of children and adolescents to commercial sexual exploitation, being worthy of mention that the sex market has not slowed down or ceased its functioning. This reality calls for State-driven measures to face such violence; however, its suppression demands the eradication of every form of inequality in this social order, making it necessary to create a new societal model.

Keywords: commercial sexual exploitation of children and adolescents, social question, pandemic capital.

\section{Introducción}

Este artículo tiene como tema la reflexión acerca de la explotación sexual comercial de niñas, niños y adolescentes en Brasil y en Paraguay en tiempos de capital pandémico.

La pandemia de COVID-19 asola el planeta oficialmente desde marzo de 2020, cuando fue reconocida por la Organización Mundial de la Salud (OMS); llega en tiempos de una coyuntura marcada por la crisis estructural del capital', que provocó el agravamiento de la "cuestión social" en virtud de la reestructuración productiva y la expansión del ideario neoliberal, que reestructuraron el Estado moderno burgués a través de una nueva disciplina presupuestaria, con abertura al capital extranjero, privatizaciones de bienes públicos, reformas fiscales, fondos públicos y financierización del capital, políticas de contención de gastos, desguace de los servicios sociales públicos, mercantilización de los derechos sociales, remilitarización de lo social, promoviendo la judicialización de las esferas sociales (Iamamoto, 2010; Yazbek, 2009).

Frente a este escenario, el COVID-19 prolifera intensamente, cobrando víctimas en la población mundial con altos números de mortandad, habiendo causado hasta el

1 Coincidiendo con la afirmación de Netto (2012), las crisis son marcas del sistema capitalista, por lo que siempre es estructural.

2 Según Iamamoto y Carvalho (1983), la “cuestión social no es más que las expresiones de la clase trabajadora y su entrada en el escenario político de la sociedad, exigiendo su reconocimiento como clase por parte de la comunidad empresarial y del Estado. Es la manifestación en la vida social cotidiana de la contradicción entre proletariado y burguesía” (p. 77) [traducción del autor] día 21 de junio de 2021 3,884,89o de muertes en el planeta, de lo que se puede destacar que, desde mayo de 2020, América del Sur es considerada uno de los epicentros de la pandemia, siendo Brasil uno de los países con mayor número de muertes, teniendo hasta la fecha referida 501,918 óbitos. En junio de 2021 se destaca el aumento de los índices de mortalidad en Paraguay, que llegan al día 21 de junio a los 11,633 óbitos (World Ometers, 2021), lo que es un número muy expresivo al considerarse la población del país.

La pandemia ha cobrado numerosas víctimas entre la población no apenas en cuestión de mortandad (lo que no es poco), sino también por las alteraciones en las relaciones sociales, al haber agravado aún más las desigualdades sociales estructurantes, alcanzando y castigando brutalmente la clase-que-vive-del-trabajo 3 .

En virtud de ello, este período es definido como "capital pandémico" por Antunes (2020, p. 21), pues, más allá de mantener y acentuar los cambios en el mundo del trabajo que ya estaban en curso en la sociedad capitalista contemporánea, con la ampliación del desempleo, la uberización ${ }^{4}$, la precarización,

\footnotetext{
3 Con base en Antunes (1999), en este artículo se utiliza la expresión "clase-que-vive-del-trabajo", ya que la composición de la clase trabajadora actual es notablemente más amplia, compleja, fragmentada y heterogénea, en comparación con la de la clase trabajadora del siglo XIX, clase, que Marx se propuso estudiar.

4 Según Antunes (2020), la uberización del trabajo se refiere al "[...] mundo del trabajo que se expande y se desarrolla en la era de la información de las plataformas y aplicaciones digitales" (p. 11) [traducción del autor].
} 
flexibilización e intensificación del trabajo, llevó a que la clase-que-vivedel-trabajo, especialmente las mujeres, poblaciones negras e indígenas, así como la población LGBTQIA+ quedasen más expuestas a la letalidad del virus SARS$\mathrm{CoV}-2$ para mantener la economía nacional e internacional, como para mantener también su propio trabajo, sea este formal $o$ informal.

Frente a todo esto es necesario destacar que esta coyuntura opera también en la vida cotidiana de niñas, niños y adolescentes, al elles no estar inmunes al proceso de las relaciones contradictorias y antagónicas del capital y del trabajo, ya que la mortalidad infantil, la desnutrición infantil, la situación de calle, la precarización educativa y la explotación sexual de niñas, niños y adolescentes son expresiones de la "cuestión social".

Según la Red ECPAT en Latinoamérica (2020), la pandemia agravó la vulnerabilidad de niñas, niños, adolescentes y sus familiares, ya que esta crisis sanitaria aumentó la desigualdad, la pobreza y el hambre, incrementando un más el riesgo de que sean sometides a las diferentes modalidades de explotación sexual comercial.

Dado todo lo que fue expuesto, surgieron varias indagaciones: ¿Cuáles son las características de la explotación sexual comercial de niñas, niños y adolescentes en Brasil y en Paraguay? ¿Esta manifestación de la "cuestión social" está ocurriendo durante la pandemia?

En el intento de responder a estas indagaciones, el presente artículo se encuentra organizado en tres secciones para forma de exposición. La primera presenta la conceptualización y características de la explotación sexual comercial de niñes y adolescentes, destacando el mercado del sexo. La segunda aborda las características de la explotación sexual comercial infantojuvenil en Brasil y en Paraguay antes de la pandemia del COVID-19, destacando rasgos de las modalidades de esta violencia en cada uno de los países. En la última sección habrá una reflexión sobre la explotación sexual comercial de niñas, niños y adolescentes en tiempos de capital pandémico, pensando cómo puede haberse presentado en estos dos países. Las consideraciones finales traen algunas sugerencias para que el Estado brasileño y el paraguayo enfrenten la explotación sexual comercial de niñas, niños y adolescentes con el propósito de garantizar la protección integral de esta población.

\section{Facetas de la explotación sexual comercial deniñas, niñosyadolescentes}

La explotación sexual comercial de niñas, niños y adolescentes es una violencia sexual caracterizada por la negociación de las actividades, prácticas y servicios sexuales de esta población por uno o más adultos que obtienen, más allá de la satisfacción sexual, ganancia.

Como destaca la ECPAT (2017), esta violencia sexual involucra transacciones económicas, las cuales pueden ser en dinero, alimento, albergue y/o algún tipo de protección. Y Chejter e Isla (2018) resaltan que la especificidad de la explotación sexual comercial es la explotación económica practicada por los intermediarios que obtienen rédito.

En este sentido, se destaca que los intermediarios o explotadores ejercen las funciones de cooptación, reclutamiento, "enganche" e intermediación, lo que puede ser realizado a través de amenazas, secuestros, convencimiento, vínculos y dependencias afectivas, emocionales o económicas. Otro agente en la comercialización sexual de niñas, niños y adolescentes es el cliente.

Como explica el Grupo Luna Nueva (2011), el término cliente, a pesar de generar controversia por contener el carácter legal de la persona que compra una mercadería y/o servicio, se utiliza también en referencia a las personas que pagan para practicar actividades sexuales con niñas, niños, adolescentes o adultos que se prostituyen o son prostituídos, siendo responsables por el movimiento de toda la cadena de 
explotación sexual comercial.

Así, se indica que la comercialización sexual de niñas, niños y adolescentes se encuentra circunscrita dentro de la lógica de mercado, siendo determinada por la lógica del capital, la explotación, la oferta y demanda, incluyendo además el aspecto sexual, o sea, el mercado del sexo.

El mercado del sexo es un ramo de actividades para todos los bolsillos y consumidores, ofreciendo diversidad de servicios y especialidades, como por ejemplo la prostitución (en calles, locales nocturnos, burdeles, cabarets, bares, karaokes, salas de cine y casas de masajes); pornografía (revistas, sitios web, líneas telefónicas, películas y shows eróticos, también encontrados en sex shops); y diversos productos (farmacéuticos, indumentaria, "juguetes sexuales", tanto en sex shops como en otros establecimientos no especializados del ramo del sexo). También existe una gran variedad de personas cuyos servicios sexuales se ofrecen y que, en sí mismas, representan la objetificación de las preferencias, intereses y necesidades de quien consume; de este modo, hay en el mercado mujeres y hombres cisgénero y transgénero, tanto aquelles dentro de los patrones social y localmente impuestos como fuera de ellos (enanes, obeses, deficientes) y también una gama de edades que cubre desde personas de la tercera edad hasta adolescentes y niños. Como apunta Rostagnol (2007), las personas prostituidas en el mercado del sexo se reducen a mercadería.

De acuerdo a Faleiros (2004), el sexo con niñas, niños y adolescentes posee gran valor comercial y es una "mercadería altamente vendible y valorizada” (p. 83) [traducción del autor].

La Declaración del Congreso Mundial sobre la Explotación Sexual de Niños realizada en Estocolmo en 1996 destaca que les niñas, niños y adolescentes víctimas de esta violencia son tratados como mercadería y objeto sexual.

$\mathrm{Y}$ es en esta relación de objeto y mercadería que niñas, niños y adolescentes seencuentran en el mercadodel sexo, siendo considerada también una de las peores formas de trabajo infantil, como define la Convención $\mathrm{N}^{\circ} 182$ de la Organización Internacional del Trabajo - OIT (1999) ${ }^{5}$.

$\mathrm{La}$ operacionalización de la explotación sexual comercial de niñas, niños y adolescentes ocurre a través de cinco modalidades distintas, sin embargo articuladas, las cuales son: prostitución infantil, pornografía infantil, turismo sexual, tráfico para fines sexuales y casamiento infantil o casamiento forzado (ECPAT, 2017).

Cabe destacar que niñas, niños y adolescentes no se prostituyen, sino que son explotados sexualmente, a pesar de que existen niñas y niños expuestos y comercializados sexualmente en las calles, carreteras, plazas, bares, restaurantes, casas nocturnas, gasolineras o estaciones de servicio, entre otros lugares. Es por ello que este término aún se utiliza para referirse a esta práctica como modalidad de la explotación sexual comercial. Sin embargo, para expresar mejor la realidad, la terminología adoptada aquí tiene como base instituciones y especialistas uruguayos tales como el Comité Nacional para la Erradicación de la Explotación Sexual Comercial y no Comercial de la Niñez y Adolescencia - CONAPEES (20162021), Instituto del Niño y Adolescente del Uruguay - INAU (2014), González (2012) y Purtscher (2014), que utilizan la expresión

\footnotetext{
5 El Artículo $3^{\circ}$ del Convenio № 182 de la Organización Internacional del Trabajo (1999, np) dice que: “(a) todas las formas de esclavitud o las prácticas análogas a la esclavitud, como la venta y la trata de niños, la servidumbre por deudas y la condición de siervo, y el trabajo forzoso u obligatorio, incluido el reclutamiento forzoso u obligatorio de niños para utilizarlos en conflictos armados; (b) la utilización, el reclutamiento o la oferta de niños para la prostitución, la producción de pornografía o actuaciones pornográficas; (c) la utilización, el reclutamiento o la oferta de niños para la realización de actividades ilícitas, en particular la producción y el tráfico de estupefacientes, tal como se definen en los tratados internacionales pertinentes, y (d) el trabajo que, por su naturaleza o por las condiciones en que se lleva a cabo, es probable que dañe la salud, la seguridad o la moralidad de los niños".
} 
actividades sexuales o eróticas remuneradas o con promesas de remuneración con niños $o$ adolescentes.

Por todo esto, se define la explotación sexual comercial de niñas, niños y adolescentes como una manifestación de la "cuestión social", ya que su constitución, caracterización, motivación, perpetuación y reproducción en la sociedad contemporánea son resultado de las relaciones contradictorias y antagónicas del capital y el trabajo, en vista de que genera lucro y se estructura a partir de la desigualdad de clase, de género, étnicoracial, de orientación sexual, generacional y social, frutos de la sociedad capitalista.

Sin embargo, como indica Iamamoto (1997), la "cuestión social" es "desigualdad, pero también resistencia, ya que los sujetos que vivencian las desigualdades reivindican su acceso al escenario político por medio de luchas a favor de los derechos y de su propia existencia" (p. 14).

El proceso de resistencia y de lucha como enfrentamiento a la explotación sexual comercial de niñas, niños y adolescentes tiene un hito la década de 1980, cuando el movimiento feminista, el movimiento LGBTQIA+ (en esa época llamado GLS), el movimiento negro, el movimiento feminista negro y los movimientos a favor de la defensa de niñas, niños y adolescentes en situación de calle, tanto como las asociaciones de prostitutas, comenzaron a denunciar la existencia de la entonces llamada prostitución infantil y las diversas formas de abuso sexual, exigiendo responsabilización del Estado.

A partir de esto tenemos el divisor de aguas en la protección de niñas, niños y adolescentes, que es la promulgación del instrumento de Derechos Humanos para esta población a través de la Convención Internacional de Derechos de la Infancia ${ }^{6}$ organizada por la Asamblea General de

6 Es de destacar que, en esta concepción de esta Convención, toda persona es menor dedieciochoaños como niño, sin reconocer la diversidad, pluralidad y diferencias entre las etapas de estas etapas de la vida. las Naciones Unidas en 1989, concibiendo y reconociéndoles como sujetos de derecho, ciudadanos libras y en desarrollo, estableciendo que todos los países signatarios deben asegurar el bienestar, la sobrevivencia y el desarrollo pleno de esta población a través del sistema de protección integral.

A partir de esta Convención Internacional de Derechos de la Infancia, la explotación sexual comercial de niñas, niños y adolescentes fue considerada una violación de los derechos humanos, pues existe un trato como "cosa", objeto y mercadería y no como sujetos libres, racionales, conscientes y de derechos.

Cabe destacar que los derechos de les niñas, niños y adolescentes tienen su base en la Declaración de los Derechos Humanos, la cual, como sostiene Barroco (2008), es un hito de la modernidad $\mathrm{y}$, consiguientemente, un avance en la genericidad humana, ya que orienta las acciones y políticas con base en la emancipación humana, lo que la convierte en una victoria de la clase trabajadora.

No obstante, inclusive con la conquista de los derechos humanos de la niñez y adolescencia y la propuesta mundial de sistemas de protección social para esta población, la comercialización sexual de niñas, niños y adolescentes continúa ocurriendo en todo el planeta, siendo también una realidad en Brasil y en Paraguay.

\section{La explotación sexual comercial de niñas, niños y adolescentes en Brasil y en Paraguay}

La explotación sexual comercial de niñas, niños y adolescentes se encuentra presente en toda la extensión territorial de Brasil y de Paraguay, habiendo semejanzas y particularidades en cada uno de los países, como también una relación directa entre ellos; está claro que existe un vínculo y una negociación del mercado del sexo que rompen fronteras geográficas.

En ambos países se practican las cinco modalidades de la explotación sexual 
Alves. No se suspende: Explotación Sexual Comercial de la Niñez y la Adolescencia en Tiempos de Capital Pandémico en la realidad brasileña y paraguaya

comercial de niñas, niños y adolescentes, siendo que las más comunes son las actividades sexuales o eróticas remuneradas o con promesas de remuneración y la pornografía infantil.

El mapeamiento de las carreteras federales brasileñas realizado entre 2017 y 2018 en territorio brasileño detectó 2.487 puntos vulnerables en todo el territorio nacional (Brasil, 2019). En Paraguay se estima que dos de tres personas que se prostituyen en el país son niñas, niño o adolescentes, con edad inicial de entre 12 y 13 años, como destaca el Grupo Luna Nueva (2011).

Se destaca en ambos países la diversidad de puntos y localidades que ofrecen actividades sexuales comerciales con niñas, niños y adolescentes, tales como: calles, plazas, áreas comerciales, gasolineras, puestos policiales, escuelas, puntos de carga y descarga, terminales de colectivos y ómnibus, puntos de alimentación, lugares con obras de arte, patios de parada de transporte, bares, casas de shows, puntos de hospedaje (hoteles, moteles, posadas, entre otros) y residencias particulares.

A respecto de la pornografía infantil, la Comisión Parlamentaria de Investigación - CPI intitulada CPI "Explotación sexual de Niños y Adolescentes de la Cámara de Diputados" de 2014, por medio de declaraciones del presidente de la ONG Safernet, Thiago Tavares Nunes de Oliveira, apunta que fueron recibidas en la institución brasileña en los últimos seis años 1.263.253 denuncias anónimas sobre páginas y sitios de Internet que mostraban niñas, niños y adolescentes expuestos sexualmente (Brasil, 2014). Por su parte, en Paraguay, la ECPAT (2014) muestra que, tan sólo en 2007, fueron encontradas 50 filmaciones con niñas, niños y adolescentes en situaciones pornográficas y 1.390 fotos con niñas desnudas junto a un hombre que producía tales materiales. Este hombre reclutaba las víctimas de pueblos empobrecidos del interior del país a través de promesas de estudio y trabajo, llevándolas a su casa en la periferia de Asunción.

Se apunta al turismo sexual como una realidad existente en ambos países; sin embargo, como indica ECPAT (2014), no existe información y datos específicos. A pesar de ello, esta institución señala que en Brasil hay fuertes indicios de que ocurra "sobre todo en las áreas de playa y costeñas del noreste brasileño, destino de turistas que vienen generalmente de Europa y de América del Norte" (ECPAT, 2014, p. 12) [traducción del autor].

El tráfico de niñes y adolescentes con fines sexuales en Brasil y Paraguay ocurra tanto en el ámbito interno como internacional, puesto que ambos países son origen, tránsito y destino. En el tráfico interno, la investigación "Tráfico de Mujeres, Niños y Adolescentes para Fines de Explotación Sexual Comercial en Brasil" de 2002 identificó 110 rutas relacionadas al tráfico interno y 131 al internacional (Leal \& Leal, 2003). Las rutas del tráfico internacional de niñas, niños y adolescentes para fines sexuales en Brasil están formadas por los países limítrofes, de los cuales se destacan Argentina, Bolivia, Guyana Francesa, Paraguay, Perú, Surinam y Venezuela; sin embargo, se encuentran también los países del continente europeo, predominando países como España, Italia, Holanda y Alemania.

En Paraguay, el tráfico internacional de niñas, niños y adolescentes para fines sexuales cuenta predominantemente con Brasil, Argentina y Perú, aunque se destaca también España como principal destino europeo; sin embargo, también lo son Italia, Alemania y Suiza e inclusive algunos países asiáticos, como Hong Kong (Grupo Luna Nueva, 2005).

Además, se detectó también es Paraguay, aunque en una escala menor, tráfico para fines sexuales con carácter matrimonial, así como matrimonio para el tráfico. El primero se refiere a personas que son traficadas para casarse y el segundo involucra personas que se casan y son traficadas posteriormente para realizar 
actividades sexuales comerciales con otro (Grupo Luna Nueva, 2005).

La otra modalidad presente en la realidad de estos dos países es el casamiento infantil, sobre el cual, a pesar de ser histórico, no existen datos oficiales. Sin embargo, según Plan International Brasil (2009), Brasil ocupa en la actualidad el cuarto lugar en el ranking internacional de uniones con niñas. Por otro lado, el Mapa de la Violencia Sexual Hacia Niñas, Niños y Adolescentes NNA7, elaborado con datos organizados a partir de casos denunciados ante la Fiscalía General del Estado duranteel período 2017-2020, indica que en Paraguay los departamentos con mayor índice de desprotección para el casamiento infantil son Boquerón, Asunción y Canindeyú. Esta violencia se encuentra naturalizada en las relaciones sociales de ambos países, ya que es considerada como una supuesta "lógica de protección”, “seguridad”, pudiendo inclusive mantener una cierta reputación y/o estatus social.

Cabe destacar que el tráfico para fines sexuales y las actividades sexuales o eróticas remuneradas o con promesas de remuneración con niñas, niños y adolescentes están vinculados en ambos países, aunque en Paraguay la puerta de entrada para estas dos modalidades es el "criadazgo", pues, según el Grupo Luna Nueva (2005), casi 90\% de las adolescentes víctimas de explotación sexual atendidas por la organización tuvieron experiencias iniciales como "criaditas". Más allá, Caputo y Cárdena (2019) destacan que los prostíbulos del país son vivificados por el llamado "criadazgo".

La mayoría de las víctimas de explotación sexual comercial es de

\footnotetext{
7 Datos consultados en el sitio web: https://www. cdiaobserva.org.py/indice-de-desproteccion/

8 Según o Grupo Luna Nueva (2005), el criadazgo "es una práctica muy extendida en Paraguay que consiste en la acogida temporal de un niño o una niña por una familia, con el fin de contribuir a mejorar sus condiciones de vida y educación, y que conlleva normalmente una contraprestación de éste en concepto de ayuda en las tareas domésticas" (p. 77).
}

género femenino, tanto cisgénero como transgénero; sin embargo, esto no significa que no haya víctimas del género masculino, sólo que esto aún está invisibilizado. En Brasil, el mapeamiento de carreteras federales y de puntos vulnerables a la explotación sexual comercial de niñas, niños y adolescentes realizado entre 2017 y 2018 identificó que $48 \%$ de las víctimas eran niñas, $36 \%$ niños y $16 \%$ transgénero (transexuales femeninas o travestis). Y en Paraguay, el Grupo Luna Nueva (2011), a partir de uma investigación por BECA y Moreno en los años 200o, destaca que fueron cuantificados 619 niñas, niños y adolescentes explotados sexualmente en las calles de Asunción, Hernandarias y Ciudad del Este, siendo $71 \%$ del sexo femenino y $29 \%$ del sexo masculino.

Además de género, la explotación sexual comercial de niñas, niños $y$ adolescentes en Brasil y en Paraguay tiene raza-color, ya que, como destaca Ecpat (2014, p.27), esta violencia está marcada por el "sesgo racial de la violencia sexual y de género" [traducción del autor]. En Brasil, las niñas negras representan la mayoría de las víctimas; como fuera identificado en el informe de 2019 del programa de denuncias Disque Derechos Humanos, conocido como Disque 100, las niñas y adolescentes negras ${ }^{9}$ son las más victimadas por las diversas formas de violencia sexual (incluyendo la explotación sexual), siendo que del total de 18.648 denuncias de situaciones de violación sexual contra niñas y adolescentes en ese año, 6.349 son de piel parda, seguidas por la piel blanca con 5.695, 1.436 negras, 97 de piel amarilla y 70 de piel indígena (Brasil, 2020).

En Paraguay, a pesar de no haber investigaciones ni datos específicos sobre la cuestión racial-étnica de las víctimas de explotación sexual comercial, se puede destacar la presencia considerable de

\footnotetext{
9 Cabe señalar que el Instituto Brasileño de Geografía y Estadística - IBGE analiza étnicamente a la población negra por la configuración de personas morenas y negras (Petruccelli e Saboiana, 2013).
} 
indígenas, siendo la situación de esta población una preocupación nacional, como destaca Dure (2011).

No obstante, en ambos países existe una valorización en el mercado del sexo en términos de lucratividad; rentabilidad y proyección son de la población blanca, pues Alves y Rodrigues (2021) explicitan que niñas, niños y adolescentes de etnia negra reciben menos por actividades y servicios sexuales realizados, comparando con aquellas de raza blanca. Además, se encuentran en el mercado del sexo en mayor riesgo y peligro de muerte. El Grupo Luna Nueva (2005, p.80) llama la atención al hecho de que en el tráfico internacional para fines sexuales con destino en el continente europeo se exige que las niñas sean "blanquitas", "finitas" y "lindas".

A pesar de todo lo mencionado, la predominancia de víctimas de la explotación sexual comercial es de niñas, niños y adolescentes oriundos de la población más empobrecida, con historial de abandono y violencia sexual, como es retratado por Faleiros (2004) al hablar de Brasil y por el Grupo Luna Nueva (2005) al tratar de Paraguay.

Sin embargo, se destaca que la situación de pobreza y miseria no son condiciones sine qua non de la explotación sexual comercial de niñas, niños y adolescentes y que esta población no es más permisiva ni culturalmente más sexualizada, aunque si la más vulnerable, ya que "la gran dependencia económica de las hijas de la clase trabajadora y la insuficiencia de acceso, estos sujetos son los más alcanzados por esse fenómeno" [traducción del autor] (Pereira, 2015, p.10o).

En este sentido, no se puede negar que las relaciones sociales estructurantes en los dos países hacen que la explotación sexual comercial de niños y adolescentes tenga clase, color y género, ya que la predominancia de las víctimas tiene origen en la clase-que-vive-de-su-trabajo y las subalternizadas del género femenino (ciso trans-) y no branca (negras e indígenas); finalmente, pertenecientes a la población históricamente marginalizada y excluida de derechos a la escolarización, a la salud, a la vivienda, a la profesionalización, al mercado de trabajo, a la renta y a la economía.

El enfrentamiento a la explotación sexual comercial en Brasil y en Argentina llegó a la agenda política en la transición de la década de 1980 para la de 1990 , cuando estos países vivenciaron el final del Estado autoritario y dictatorial e iniciaron el proceso de democratización. No obstante, el ideario neoliberal entra en ambos países, debilitando la intervención del Estado, que no logra fortalecerse ni consolidarse en estos.

Es en la lucha por el proceso de democratización de los países que movimientos sociales, como por ejemplo el Movimiento Negro, el Movimiento feminista, el Movimiento LGBTQIA+, comienzan a denunciar la existencia de la explotación sexual comercial de niñas, niños y adolescentes, exigiendo al Estado que enfrente esta manifestación de la "cuestión social" y que hay a protección integral de niñas, niños y adolescentes.

En Brasil puede destacarse el Movimiento en Defensa de los Derechos del Niño y del Adolescente - MDCA, y el Movimiento Nacional de Niños y Niñas de Calle - MNMMR, que descubrieron y llamaron atención hacia la existencia de niñas en situación de calle que comercializaban la actividad sexual para sobrevivir; y de allí se pasó a pensar en la relación de la niña en situación de calle con la explotación sexual, como también en la necesidad de protección integral de la población infanto-juvenil (SANTOS, 2004). $Y$ en Paraguay, el Grupo Luna Nueva (2005) destaca la importancia del movimiento feminista en el país, que pedía la desnaturalización de la violencia contra mujeres y niñas, reclamando y denunciando tales violaciones.

Frente a estas movilizaciones, estos países se tornaron signatarios de la Convención de las Naciones Unidas de 1989 sobre los Derechos del Niño, asumiendo compromisos de implantar el sistema de 
protección de niñas, niños y adolescentes, decretando los hitos legales avanzados, identificando esta población como sujetos dederechosycomprendiendola explotación sexual comercial como violación de los derechos humanos, la Constitución Federal de 1988 y el Estatuto da Criança e do Adolescente - ECA (1990) en Brasil y la Constitución Nacional de Paraguay (1992) y el Código de la Niñez y de la Adolescencia (2001), instituyendo los consejos participativos, que tienen como función estratégica la de velar por el cumplimiento de los derechos de niñes y adolescentes, denominados en Brasil Conselho Tutelar (1990) y en Paraguay Consejerías de los Derechos del Niño - CODENI (2001) y Consejos Departamentales de la Niñez y Adolescencia (2004).

No obstante todo esto, solamente a partir de los años 2000 inician las primeras políticas específicas de enfrentamiento a la explotación sexual comercial de niñas, niños y adolescentes, instituyendo e implementando en Brasil el Plan Nacional de Enfrentamiento a la Violencia Sexual Infanto-Juvenil 2000, reformulado en 2013. En Paraguay se desarrolló el Plan Nacional de lucha contra la ESNNA (2003 y 2008); sin embargo, como destaca el Grupo Luna Nueva (2011), este no fue implementado formalmente, siendo revisado en 2010 y la Ecpat (2014b) menciona que este plan fue reformulado, actualizado y rebautizado, siendo desarrollado el Plan Nacional de Prevención y Erradicación de la Explotación Sexual de Niñas, Niños y Adolescentes (2012-2017).

A pesar de avances en estas últimas tres décadas a favor de la defensa de niños y adolescentes y de las medidas de enfrentamiento a la explotación sexual comercial realizadas en estos países, se identifica que esta manifestación de la "cuestión sexual" continúa presente en la realidad social brasileña y paraguaya, violentando niñas, niños jóvenes $y$ adolescentes transexuales.

\section{Explotación sexual comercial de niñas, niños y adolescentes en tiempo de capital pandémico}

Durante la pandemia de COVID-19 el mercado del sexo no adhirió al aislamiento y el distanciamiento social, paralizando sus acciones, como fuera recomendado para todas las actividades productivas y relaciones sociales por la Organización Mundial de la Salud y las autoridades sanitarias, siendo tales medidas la única posibilidad de achatamiento de la curva de contaminación, evitando de esta manera la propagación mayor del virus.

Consecuentemente, el mercado del sexo continúa operando en diversos lugares y de diferentes formas; al final, como con otras actividades económicas, lo importante es el lucro y estas medidas de salud pública perjudican el capital. De este modo, niñas, niños y adolescentes quedan aún más vulnerables en este momento, en virtud del agravamiento de la "cuestión social” y también por la paralización, cierre y/o adopción de formas remotas de atención por los diversos servicios del sistema de protección social, como escuelas, instituciones socioasistenciales y jurídicas.

En Brasil, las actividades sexuales o eróticas remuneradas o con promesas de remuneración con niñes o adolescentes continuaron existiendo; la Red Peteca ${ }^{10}$, por ejemplo, informósobrela subnotificaciónde las denuncias y atención, y específicamente reveló la denuncia de seis casos de niñas y adolescentes en situación de explotación sexual comercial en la ciudad de San Pablo al inicio de la pandemia.

A respecto de la pornografía infantil, el Consejo de Derechos Humanos de las Naciones Unidas (2021), Red Ecpat en Latinoamérica (2020) y UNICEF (2020) llaman la atención hacia la mayor vulnerabilidad de niñas, niños y adolescentes para ser víctimas de esta

10 Datos consultados en el sitio web: https:// livredetrabalhoinfantil.org.br/noticias/reportagens/ subnotificada-exploracao-sexual-exige-ainda-maisatencao-da-rede-de-protecao-durante-a-pandemia/ 
modalidad, porque en tiempos de pandemia el acceso a la Internet, que ya estaba en alza en las últimas décadas, ahora aumentó aún más, tornándose una de las principales formas de interacción social, espacio de trabajo y educativo valorizado por el capital. Al final, el trabajo remoto o teletrabajo y los "influencers" o "youtubers" han asolado el cotidiano de los trabajadores.

En el período inicial de la pandemia hubo un aumento del consumo de Internet en Brasil y en Paraguay. Como apunta Akamai, una plataforma de almacenamiento en nube, en abril el aumento fue de $112 \%^{11}$ y la empresa de telecomunicaciones Tigo Paraguay destaca que el aumento del consumo de Internet en Paraguay fue de $40 \%{ }^{12}$. Esto puede hacer suponer que niñas, niños y adolescentes también están más tiempo en Internet, ya que están estudiando a través de ella; como también se encuentran jugando en este espacio virtual (lo que ya ocurría antes de la pandemia). Sin embargo, esto no es exclusividad suya: los explotadores (incluyendo los "consumidores") también están conectados.

En este sentido, la Coordinadora por los Derechos de la Infancia y la Adolescencia (CDIA) (2021) destaca que el aumento en el uso de la Internet y las nuevas tecnologías torna posibles nuevas formas e instrumentos que garantizan el anonimato de las redes de explotación, permitiendo y facilitando prácticas de violencia contra niñas, niños y adolescentes, como reclutamiento, extorsión y coerción sexual de forma online.

A respecto del tráfico para fines sexuales contra niñas, niños y adolescentes en tiempos de capital pandémico, existe como hipótesis el aumento de casos y

11 Datos consultados en el sitio web: https:// tecnoblog.net/344896/exclusivo-akamai-ve-uso-deinternet-crescer-112-no-brasil-durante-pandemia/

12 Datos consultados en el sitio web: https:// www.icex.es/icex/es/navegacion-principal/todosnuestros-servicios/informacion-de-mercados/ paises/navegacion-principal/noticias/paraguayinternet-new2020852401.html?idPais=PY situaciones, ya que el cierre de las fronteras marítimas, aéreas o terrestres tendrá como resultado un esquema aún más perverso de circulación y de contrabando de personas y materiales, como apunta el Informe de Investigación del Despacho sobre Drogas y Crímenes de la Organización de las Naciones Unidas - UNODC (2020).

En este sentido, se indican los dichos de la Ministra de la Niñez y de la adolescencia de Paraguay, Teresa Martínez, que destacan que al inicio de la pandemia fueron rescatadas más de 130 niñas, niños $\mathrm{y}$ adolescentes paraguayas en la frontera entre Brasil y Paraguay, que regresaban de la ciudad de San Pablo, Brasil, luego de quedar en situación de explotación laboral (fábricas de confecciones y servicio doméstico) y/o explotación sexual ${ }^{13}$.

Según la OIT (2020), niñas, niños y adolescentes se encuentran vulnerables en este período de crisis sanitaria, pudiendo ser más blanco del tráfico para fines sexuales, como también para otras modalidades de exploración sexual y otras formas de trabajo infantil.

En este sentido, se destaca como una de las vulnerabilidades que pueden verse acentuadas en este momento el casamiento forzado, del cual el consejo de Derechos Humanos de las Naciones Unidas (2021, p.11) estima que "para 2030, la pandemia podría dar lugar en todo el mundo a unos 13 millones más de matrimonios infantiles de los que se habrían producido en otras circunstancias".

Esta modalidad puede de hecho ocurrir de forma agravada en este coyuntura, ya que está asociada histórica y culturalmente en Brasil y en Paraguay a una supuesta "seguridad" de la persona menor de edad, como también de las familias, siendo que las relaciones de intercambios comerciales y sexuales están vinculadas a una "protección", la cual

13 Datos consultados en el sitio web: https://www. lanacion.com.py/pais/2021/o3/23/pandemia-2020detectaron-135-menores-paraguayos-victimas-deexplotacion-y-trata-en-brasil/ 
puede ser vivienda, alimentación, ropa, tierra, aparatos electrónicos, como también honra, reputación y prestigio social.

La única modalidad de explotación sexual comercial de niñas, niños $y$ adolescentes que puede haber disminuido en este período de capital pandémico es el turismo sexual, ya que como divulgó la Organización Mundial del Turismo OMT $^{14}$ el pronóstico de caída de viajes internacionales en todo el mundo; o sea, puede suponerse que habiendo menos gente viajando, consecuentemente se haya reducido la cantidad de turistas dispuestos a violentar niñas, niños y adolescentes.

Sin embargo, como señala el Consejo de Derechos Humanos de las Naciones Unidas (2021), las restricciones de viajes internacionales han provocado un aumento en el tráfico interno de niñas, niños y adolescentes, como también han generado una mayor demanda de niñas, niños y adolescentes locales.

Basándonos en esto se destaca que el mercado del sexo con niñas, niños y adolescentes también ha cambiado su dinámica para atender la demanda y la necesidad del "cliente", incorporando y adoptando nuevas formas de comercializar las actividades sexuales; como cita el Consejo de Derechos Humanos de las Naciones Unidas (2010), se há observado la venta y las actividades sexuales con niñas, niños y adolescentes dentro de vehículos, en las redes sociales y a través de aplicaciones de mensajería.

La realidad presentada aquí desafía y pide la intervención del Estado en la protección de niñas, niños y adolescentes y, por consiguiente, en el enfrentamiento de la explotación sexual comercial. Sin embargo, no se han identificado acciones concretas y efectivas en ninguno de los países para combatir la explotación sexual en tiempos de capital pandémico.

14 Datos consultados en el sitio web: https:// www.cndportugues.com/pt-br/turismo/turismointernacional-altas-perdas-durante-quarentenapela-covid-19

\section{Consideraciones Finales}

Este artículo pone en evidencia que la pandemia de COVID-19 agravó aún más la crisis del capital contemporánea, reforzando las desigualdades sociales, afectando las condiciones de sobrevivencia de la población que vive del trabajo, que es históricamente marcada por la desprotección social en estos países, intensificando la vulnerabilidad de niñas, niños y adolescentes, la cual ya era alarmante en ambas naciones.

Además, se destaca que las redes de explotación sexual comercial en Brasil y en Paraguay son organizadas, pero la red de protección de la niñez y la adolescencia tiene mucho por hacer para organizarse.

Lo primero que ha de ser hecho es que el Estado en estos países se responsabilice por la protección de niñas, niños y adolescentes y sus familiares. Al final, es su obligación crear medidas y acciones para combatir la comercialización sexual de niñas, niños y adolescentes; recordando también que tanto Brasil como Paraguay son signatarios de la Convención de los Derechos de los Niños de las Naciones Unidas de 1989, la Declaración y Agenda para la Acción contra la explotación sexual comercial de niñas, niños y adolescentes de Estocolmo de 1996, la Convención № 182 de la OIT sobre Prohibición de las Peores Formas de Trabajo Infantil y Acción Inmediata para su Eliminación de 1999, y la Convención de Palermo de 2004. Además, los Estados precisan ejecutar la legislación y los planes nacionales por medio de políticas sociales desarrolladas, siendo que ellos fueron construidos por profesionales especializados de diversas áreas, demostrando la importancia de la ciencia, la investigación y una formación calificada; pues sólo de este modo puede pensarse en políticas sociales de acuerdo con la realidad y la demanda social, siendo por lo tanto fundamental la ciencia para el enfrentamiento de la exploración sexual comercial niñas, niños y adolescentes.

Así, se sugiere como posibilidades acciones para el enfrentamiento de la 
explotación sexual comercial en este período de pandemia para la prevención e identificación de situaciones de riesgo y la consiguiente búsqueda de auxilio pautadas en la esfera del sistema educacional, ya que es importante que la política educativa aborde la cuestión de la violencia sexual, los derechos de niñas, niños y adolescentes, los derechos sexuales y reproductivos, como también los debates sobre color-razaetnia y la cuestión de género (identidad y dominación). Esta cuestión urge en el actual escenario de aislamiento social, en el cual la educación viene siendo realizada a través de la enseñanza remota, donde niñas, niños y adolescentes no van a la escuela, dificultando la percepción de los educadores sobre el cotidiano de esta población; de ahí que la autoprotección deba ser reforzada.

El proceso educativo no se realiza solamente en la escuela, sino también fuera de ella, siendo la familia y la comunidad también responsables. Sin embargo, para esto ellas precisan ser educadas e informadas, para estar entonces también comprometidas y movilizadas. En tiempos de pandemia, esto tiene que ser reforzado, siendo hecho con rigor, pudiendo utilizarse herramientas tecnológicas actuales.

De este modo se sugiere también la realización de campañas de "conscientización" de la población, pues, a pesar de que la explotación sexual comercial permea la cotidianeidad, esta es aun invisible en relación a la participación de la sociedad civil en el enfrentamiento, en la protección y en la percepción de niñas, niños y adolescentes como víctimas, ya que a lo largo de nuestra historia fueron tachados de prostitutos, marginales $y$ provocadores de esta situación.

Otraacción quedebeser realizada es la creación de más servicios socioasistenciales para atender y abrigar niñas, niños y adolescentes víctimas y sus familiares, ya que incluso antes de la pandemia ya existía desfasaje en ambos países en cantidad de servicios. En función de esto, es necesario contar con más inversión material y personal, posibilitando la atención de las diversas demandas de la población.

Además, es necesario que se creen servicios de atención a los agresores"clientes", ya que quien cometió la violencia fueron ellos, y este ciclo tiene que terminar. En vista de eso, ellos tienen que estar involucrados en este proceso de atención, más allá de la medida punitiva.

También se necesita interseccionalidad de políticas sociales; al final, la responsabilidad por el enfrentamiento a la explotación sexual comercial de niñas, niños y adolescentes es de orden de las políticas de educación, de asistencia social, de salud, de cultura, de seguridad pública, de justicia, de deporte y educación, siendo todos ellos responsables por la protección integral de esta población. En virtud de esto, en este momento de pandemia se hace necesaria la ampliación de estos servicios con recursos materiales y personales.

De este modo, se refuerza aquí que la protección social pública no puede ser tratada como gastos, pues garantizar atención, prevención y atención a niñas, niños adolescentes y sus responsables es inversión; por eso el camino es la ampliación de los servicios públicos y el acceso a los derechos.

Otra acción a ser tomada es la realización de relevamiento diagnóstico, y esto compete a los profesionales de los ámbitos transversales de las políticas sociales, pues permite conocer los lugares de concentración de niñas, niños y adolescentes - en especial en situación de vulnerabilidad - tales como: lugares de venta y uso de drogas y de tráfico, puntos de prostitución y situación de calle. Como ya fuera explicitado, el mercado del sexo durante la pandemia continúa existiendo; entonces, su mapeamiento es necesario.

También como sugerencia, se precisa fortalecer el proceso de protagonismo de niñas, niños y adolescentes, porque tienen mucho para decirnos, ya que son sujetos de su propia historia, teniendo sueños, angustias, deseos, experiencias diversas, 
así como también varias preguntas, indagaciones y respuestas. Este proceso de protagonismo contribuye para que se vislumbren otros procesos de sociabilidad que no atiendan exclusivamente la lógica del capital.

Finalmente, la eliminación de la explotación sexual comercial de niñas, niños y adolescentes sólo será posible cuando sean establecidas acciones que busquen romper con el modelo social pautado en el sexismo, el machismo, el racismo, el clasismo, la discriminación generacional, territorial y la explotación, formulando un proyecto social con base en la emancipación humana y social.

\section{Referencias}

Alves, A. L. \& Rodrigues, A. S (2021). Exploração Sexual Comercial InfantoJuvenil em Questão: social, racial e de gênero. En: A. S. Rodrigues (Ed.), Serviço Social e debates contemporâneos: Trilhando reflexões para o trabalho e a formação profissional. Editora CRV.

Antunes, R. (1999). Os sentidos do trabalho - ensaio sobre a afirmação e negação do trabalho. Boitempo.

Antunes, R. (2020). Coronavírus: o trabalho sob fogo cruzado. São Paulo: Boitempo (ebook).

Barroco, M. L (2008). O significado sóciohistórico dos direitos humanos e o Serviço Social. CFESS. http://www. cfess.org.br/pdf/maria_lucia_barroco. pdf

Brasil. (2019). Mapeamento dos pontos vulneráveis à exploração sexual de crianças e adolescentes nas rodovias federais brasileiras. 8a. https://www. chegadetrabalhoinfantil.org.br/wpcontent/uploads/2019/o4/MapearCartilha.pdf

Brasil. (2020). Disque direitos humanos: Relatório 2019. Ministério da Mulher, da Família e dos Direitos Humanos.

Brasil. (2014). Comissão Parlamentar de Inquérito da Exploração Sexual de
Crianças e Adolescentes. Câmara dos Deputados. https://www2.camara.leg. br/atividade-legislativa/comissoes/ comissoes-temporarias/parlamentarde-inquerito/54a-legislatura/cpiexploracao-sexual-de-criancas-eadolescentes/relatorio-final-aprovadol atrioaprovadoVERSOFINALcomauten ticao.pdf

Caputo, L. \& Cárdenas, M. S. (2019). Trata de personas labora y sexual de niños, niñas y adolescentes. Buenas intenciones no alcanzan, el Estado deve actuar de verdad. En Yvypóra Derécho Paraguáipe - Derechos Humanos en Paraguay 2019. Codehupy.

Chejter, S. \& Isla, V. (2018). Abusos sexuales y embarazo forzado hacia niñas, niños y adolescentes Argentina, América Latina y el Caribe. Hoje informativa. https://argentina.unfpa. org/sites/default/files/pub-pdf/ HojaInformativa_ENIA.pdf

CONAPESS. II (2016). Plan Nacional para la erradicación de la explotación sexual de niñas, niños y adolescentes 2016-2021: 100 acciones acordadas. CONAPEESINAU. https://tbinternet.ohchr.org/ Treaties/CAT/Shared\%2oDocuments/ URY/INT_CAT_ADR_URY_3180o_S.pdf

Consejo de Derechos Humanos das Nações Unidas (2021). Efectos de la enfermedad por coronavirus en las diferentes manifestaciones de la venta y la explotación sexual de niños. EN: Informe de la Relatora Especial sobre la venta y la explotación sexual de niños, incluidos la prostitución infantil, la utilización de niños en la pornografía y demás material que muestre abusos sexuales de niños, Mama Fátima Singhateh. $46^{\circ}$ período de sesiones 22 de febrero a 19 de marzo de 2021. https:// reliefweb.int/sites/reliefweb.int/files/ resources/A_HRC_46_31_S.pdf

Coordinadora por los Derechos de la Infancia y la Adolescencia (CDIA). (2021). Paraguay muestra alto riesgo de violencia y explotación sexual de niñas, niños y adolescentes. https://www. cdia.org.py/2021/05/31/alto-riesgoviolencia-y-explotacion-sexual-nna 
Dure, E. (2011). La trata interna de niñas, niños y adolecentes con fines de explotación sexual. Características y factores que inciden. Luna Nueva. http://www.vuelalibre.info/wpcontent/uploads/2019/o4/2_Informe_ trata-interna-1.pdf

ECPAT. (2017). Relatório de Monitoramento de País sobre Exploração Sexual Comercial de Crianças e Adolescentes. ECPAT. http://ecpatbrasil.org.br/ site/wp-content/uploads/2019/o6/ Monitoramento-de-Pa\%C3\%ADsECPAT-2017.pdf

ECPAT.(2014a).Relatório de Monitoramento de país sobre a exploração sexual comercial de crianças e adolescentes ESCCA Brasil. ECPAT Internacional. https://www.ecpat.org/wp-content/ uploads/2016/04/CMR_BRAZIL_ FINAL.pdf

ECPAT. (2014b). Informe de Monitoero de Papis sobre la Explotación Sexual Comercial de Niños, Niñas y Adolescentes. Paraguay. https:// www.ecpat.org/w p-content/ uploads/2016/04/CMR_PARAGUAY_ FINAL.pdf

ESTOCOLMO. (1996), Declaración y programa de acción - Primero Congreso Mundial Contra La Explotación Sexual Comercial de los Niños. Estocolmo, Suécia. http://white.lim.ilo.org/ipec/ documentos/decla_estocolmo.pdf.

Faleiros, E. T. S (2004). A exploração sexual comercial de crianças e adolescentes no mercado do sexo. En: R. M. C. Libório, Renata Maria e S. M. G. Sousa, Sônia M. Gomes (Eds.). A exploração sexual de crianças e adolescentes no Brasil. Reflexões teóricas, relatos de pesquisas e intervenções psicossociais. Casa do Psicólogo.

González, D. (Ed.) (2012). Explotación y desamparo 2012: Informe sobre la explotación sexual comercial de niñas $y$ adolescentes mujeres en Uruguay. Montevideo, Uruguay: Asociación Civil de Servicios y Acciones por la Infancia. Fundación Justicia y Derecho. http://observatoriojudicial.org.uy/ wp-content/uploads/2019/o4/2012Explotacion-y-Desamparo_web.pdf

Grupo Luna Nueva. (2011). Construyendo una estrategia de supervivencia que no las lleve a la calle. Proyecto "Promoción de los derechos de niñas y adolescentes en situación de explotación sexual en Paraguay". http://www.vuelalibre. info/wp-content/uploads/2019/o4/11_ Construyendo-una-estrategia-desupervivencia-que-no-las-lleve-a-lacalle.pdf

Grupo Luna Nueva (2005). La Trata de Personas en el Paraguay: Diagnóstico exploratorio sobre el tráfico y/o trata de personas con fines de explotación sexual. Organización Internacional para las Migraciones. https:// publications.iom.int/system/files/pdf/ la_trata_personas_paraguay.pdf

Iamamoto, M. V. (1997). O Serviço Social na Contemporaneidade: Trabalho $e$ Formação Profissional. Cortez.

Iamamoto, M. V (2010). Serviço Social em tempo de capital fetiche: capital financeiro, trabalho e questão social ( $4^{\mathrm{a}}$ ed.). Cortez.

Iamamoto, M. V. \& Carvalho, R (1983). Relações Sociais e Serviço Social no Brasil. Cortez.

INAU. (2014). Modelo de atención de INAU, para situaciones de explotación sexual comercial infantil y trata de niñas, niños $y$ adolescentes con fines de explotación sexual. Uruguay.

Leal, M. L. P. \& Leal, M. F. (Ed.). (2003). Pesquisa sobre tráfico de mulheres, crianças e adolescentes para fins de exploração sexual comercial no Brasil. Relatório Nacional. CECRIA.

Netto, J. P (2012). Crise do capital e consequências societárias. Revista Serviço Social e Sociedade, São Paulo, (111). https://www.scielo.br/pdf/sssoc/ n111/a02.pdf

OIT. (1999). Convenção sobre Proibição das Piores Formas de Trabalho Infantil e Ação Imediata para sua Eliminação Convenção № 182. http://www.tst.jus. br/documents/2237892/ 
OIT. (2020). Precisamos protegeras crianças do trabalho infantil. https://www.ilo. org/brasilia/noticias/WCMS_748018/ lang--pt/index.htm.

Pereira, A. A. (2015). Exploração Sexual: a condição de vida das filhas da classe trabalhadora. [Mestrado em Serviço Social] - Pontifícia Universidade Católica de São Paulo.

Petruccelli, J. L. \& Saboia, A. L. (Ed.). (2013). Características Étnico-Racias da População: Classificações e Identidades. IBGE.

Plan International Brasil. (2019). Tirando o véu. Estudo sobre casamento infantil no Brasil. https://plan.org.br/wpcontent/uploads/2019/o6/EstudoCasamento-Infantil-Brasil_final.pdf

Purtscher, L. (Ed.). (2014). Um secreto a vocês. INAU, Uruguay. https:// www.vozyvos.org.uy/wp-content/ uploads/2015/o9/un-secreto-a-voces_ webi.pdf

Red ECPAT en Latinoamérica (2020). Llamado a proteger a las niñas, niños y adolescentes de la explotación sexual comercial en Latinoamérica en el marco de la pandemia por COVID-19. https://www.ecpat.org/wp-content/ uploads/2020/o7/LLAMADO-APROTEGER-A-LAS-NIN\%CC\%83ASNIN\%CC\%83OS-Y-ADOLESCENTESEspanol.pdf

Rostagnol, S (2007). Historias en el silencio: prostitución infantil y adolescente en Montevideo y área metropolitana. http://www.bibliotecaunicef.uy/doc num.php?explnum_id=44
Santos, B. R. (2004). Contribuições para um balanço das campanhas de combate ao abuso e exploração sexual de crianças e adolescentes no Brasil. Em R. M. Libório, R. M. C., S. M. G. Sousa. (Ed.), A exploração sexual de crianças e adolescentes no Brasil. Reflexões teóricas, relatos de pesquisas e intervenções psicossociais. Casa do Psicólogo.

UNICEF. (2020). Declaración de Henrietta Fore, Directora Ejecutiva de UNICEF. No dejemos que los niños sean las víctimas ocultas de la pandemia de COVID19. https://www.unicef.org/ argentina/comunicados-prensa / no-dejemos-ninosvictimas-ocultaspandemia-covidig.

UNODC. (2020). Research and Trend Analysis Branch, Vienna - Austria, 2020. How COVID-19 restrictions and the economic consequences are likely to impact migrant smuggling and crossborder trafficking in persons to Europe and North America. https://reliefweb. int/sites/reliefweb.int/files/resources/ Covid-related-impact-on-SoM-TiPweb3.pdf

Yazbek, M. C (2009). O significado sóciohistórico da profissão. In: Serviço Social Direitos Sociais e Competências Profissionais. CFESS/ABEPSS.http:// unesav.com.br/ckfinder/userfiles/ files/O_significado_socio-_historico_ da_profissao\%2oYasbek.pd $\bar{f}$

\section{Sobre el Autor}

Alan de Loiola Alves

Asistente Social, Especialista en Atención de Niños y Adolescentes Víctimas de Violencia Doméstica (Pontificia Universidad Católica de Rio de Janeiro PUC-Rio), Magister en Servicio Social (PUC-Rio). Estudiante de Doctorado en Servicio Social en la Pontificia Universidad Católica de San Pablo PUCSP. Investigador del Núcleo de Estudos e Pesquisas sobre Crianças e Adolescentes (NCA/PUC-SP). Posee experiencia profesional en la docencia en la carrera de Servicio Social en las materias del Eje de Fundamentos Históricos Teóricos Metodológicos del Servicio Social y en atención de niños y adolescentes víctimas de explotación sexual comercial y en situación de calle. 